\title{
Religion and Mental Health During Incarceration: A Systematic Literature Review
}

\author{
Ariel Eytan
}

Published online: 11 February 2011

(C) Springer Science+Business Media, LLC 2011

\begin{abstract}
Religion and spirituality (RS) as a coping resource for facing stressful life events is encountered with increasing frequency in the medical literature. RS is associated with more favourable outcomes among people suffering from mental disorders. Detention is a stressful situation and the prevalence of mental disorders in places of detention is increased compared with the community. This literature review examined the association between RS and the mental health of detainees. PubMed, PsycINFO, Web of Science and the Internet were systematically searched from inception of each data base to August 1st 2010. Peer reviewed articles that reported primary empirical data about the impact of spirituality on the mental health and behaviour of detained persons were selected. Qualitative studies are also discussed. Twelve empirical studies including a total of 4,823 individuals met our inclusion criteria. RS is associated with lower frequency and severity of depressive episodes. The strongest reported effect of RS on prison life is a reduction of incidents and disciplinary sanctions. Prospective targeted studies are needed in order verify the hypothesis that RS reduces suicide among detainees.
\end{abstract}

Keywords Religion $\cdot$ Spirituality $\cdot$ Prison $\cdot$ Detention

\section{Introduction}

It is estimated that about 9.8 million people are held in penal institutions throughout the world. Almost half of these are in the United States, Russia or China. The US also have one of the highest prison population rates in the world with approximately 750 per 100,000 of the population being incarcerated [1]. Prisons, prison populations and juridical policies differ to a large extent from one country to another [2]. Torture or other forms of human right abuses, length of detention, status of detention (remand versus sentenced) are among

\footnotetext{
A. Eytan $(\bowtie)$

Department of Psychiatry, University Hospital of Geneva, 2 Ch. Petit-Bel-Air, 1225 Geneva,

Switzerland

e-mail: Ariel.Eytan@hcuge.ch
} 
the factors that should be taken into account when studying prisoners' mental health. Compared with the general population, prison populations are usually younger, with a predominance of males.

Several studies conducted during the last two decades have shown an increased prevalence of mental disorders among prisoners, compared with rates observed in the general population [3]. A systematic review and meta-analysis published in 2002 concluded that, typically, about one in seven prisoners in Western countries have psychotic illnesses or major depression [4]. Most prevalence studies were conducted in North America, Europe, Australia and New Zealand. For non-Western countries, reliable data is almost non-existent but there are indications suggesting that the prevalence of mental disorders in jails and prisons is similar to what is observed in the West [5-7].

Suicide is an omnipresent preoccupation in detention and numerous studies describe the increased rates of suicide that exist in jails and prisons in several countries [8]. Several studies have shown that age-standardized rates of suicide among male prisoners are between five and eight times higher than in the general population [9], and sometimes as high as fourteen times higher [10]. Suicide in prison has a strong correlation with hopelessness and the impossibility to have recourse to the usual coping mechanisms. For example, a prisoner is not usually able to participate in rituals associated with the funeral of a loved one [11]. The impact of the prison environment on social networks and availability of social support has been underlined [12]. In order to reduce the burden of mental suffering and suicide, it is important to allow or facilitate access to coping resources possibly available in places of detention. Spiritual support is often mentioned as one of them, but there is little empirical data demonstrating its real impact. Behavioural problems or hostility may also be related to mental or personality disorders. In prison, behavioural inadequacy sometimes leads to fights, victimization and periods of restraint for disciplinary motives. Here too, coping mechanisms such as religious involvement may be helpful to prevent this type of problems.

Generally speaking, and despite spectacular advances in technology and sciences, religion as a coping behaviour remains widespread. When people are confronted with severe and chronic medical conditions, religious coping is the rule rather than the exception [13]. Patients with mental disorders, ranging from anxiety to schizophrenia or depression with suicidal ideation, may also benefice from spiritual support [14]. Religion also acts as a protective factor against addictions and substance misuse [15].

The aim of the present paper is to review empirical data on religion, spirituality and mental health or illness in places of detention. A difficulty is that spirituality and religion encompass different phenomena that don't completely overlap. A certain degree of confusion between both concepts sometimes exist in the medical literature [16]. Religion involves beliefs, practices and rituals, whereas spirituality is more personal. However, as proposed by Koenig, we will use the terms religion and spirituality synonymously (referred to as RS), except if otherwise specified.

\section{Methods}

A systematic bibliographic search was undertaken from databases (PubMed, PsycINFO, Web of Science and the Internet) to indentify all articles in English or in French published from inception of each data base to August 1st 2010 that examined RS and mental health in places of detention. Key words used were religion, religiosity, spiritual, spirituality, faith, 
prison, jail, detention and detainee. From more than two hundred references, 12 met our inclusion criteria: peer reviewed articles that reported primary data about the impact of spirituality on the mental health and behaviour of detained persons. We excluded from the analysis opinion papers, published thesis, studies which dealt with RS issues only marginally and studies about RS in released detainees living in the community. Six relevant qualitative studies were retrieved and are also discussed.

\section{Results}

\section{Empirical Studies}

Results from the twelve empirical studies are presented chronologically in Table 1. Considering that 661 subjects were included in the two studies by Fernander et al. [17, 18], a total of 4,823 individuals in detention were studied. Among these, 309 (6.4\%) were females. Except for the 216 British prisoners included in the study by Adler et al. [19], all subjects were incarcerated in US facilities. Methods and questionnaires were diverse. Regarding the assessment of RS, a total of 11 structured instruments were used. RS was shown to improve coping, reduce depressive symptoms or self-harm in five studies. In 6 studies, RS had an impact on inmates' behaviour by reducing arguments, violence and disciplinary sanctions.

\section{Qualitative Data}

When examining empirical data and narratives, several specific domains emerge, such as religious conversions in prison, RS as a coping mechanism for facing incarceration and RS in the process of psychosocial rehabilitation and re-entry in the community:

Studying religious conversions during incarceration in 63 inmates, Kerley and Copes [20] observed that faith provided a motivation and focus for dealing with the pains of confinement and help them create a self-image. RS did not, however, take away the isolation, deprivation and other problems linked to the prison experience. Using a social context approach, Dix-Richardson showed that conversion to Islam among African American detained women is extremely low, compared with male counterparts [21].

Among a sample of 12 imprisoned battled women, most had what they described as spiritual experiences during their time in prison [22]. This study suggests that RS gave them a way to reconstruct and reinterpret their victimization, perpetration of violence and subsequent incarceration. Using an ethnographic methodology including participant observation and 70 interviews, Dammer discusses the reasons for religious involvement in prison [23]. According to this author, RS serves to mitigate the psychological and physical deprivations created by imprisonment. Weather the inmates are sincere or insincere in belief and practice appears to be an important issue, since they may sometimes join religious programs for the benefit of early parole release. Some others view RS in prison as a symbolic world with its own constitutive rules as being part of the global inmate culture [24]. In a study applying an ad hoc questionnaire for assessing hope after liberation in a sample 271 female detainees in Romania, spiritual values were beneficial and raised the hopes of social reintegration [25]. 


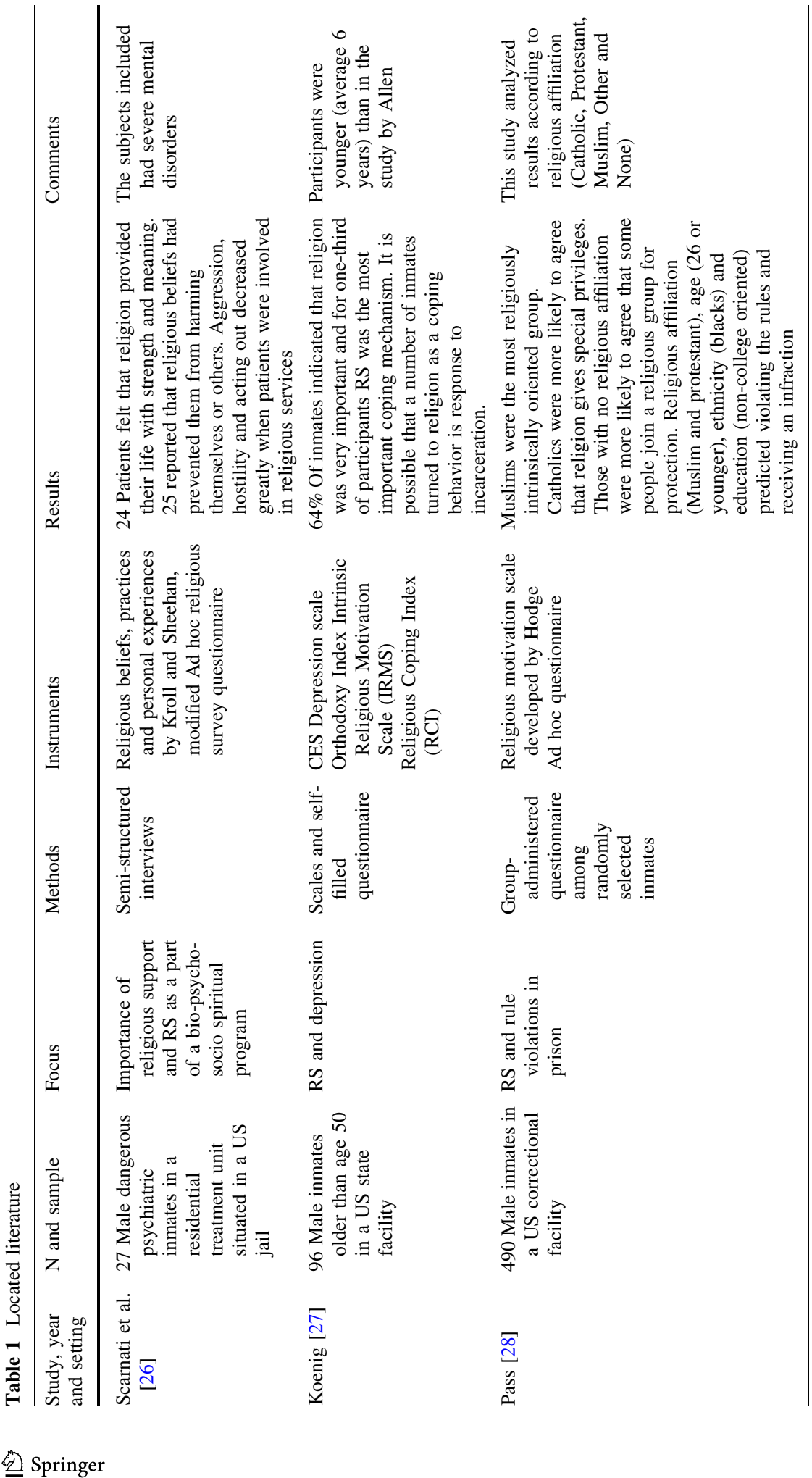




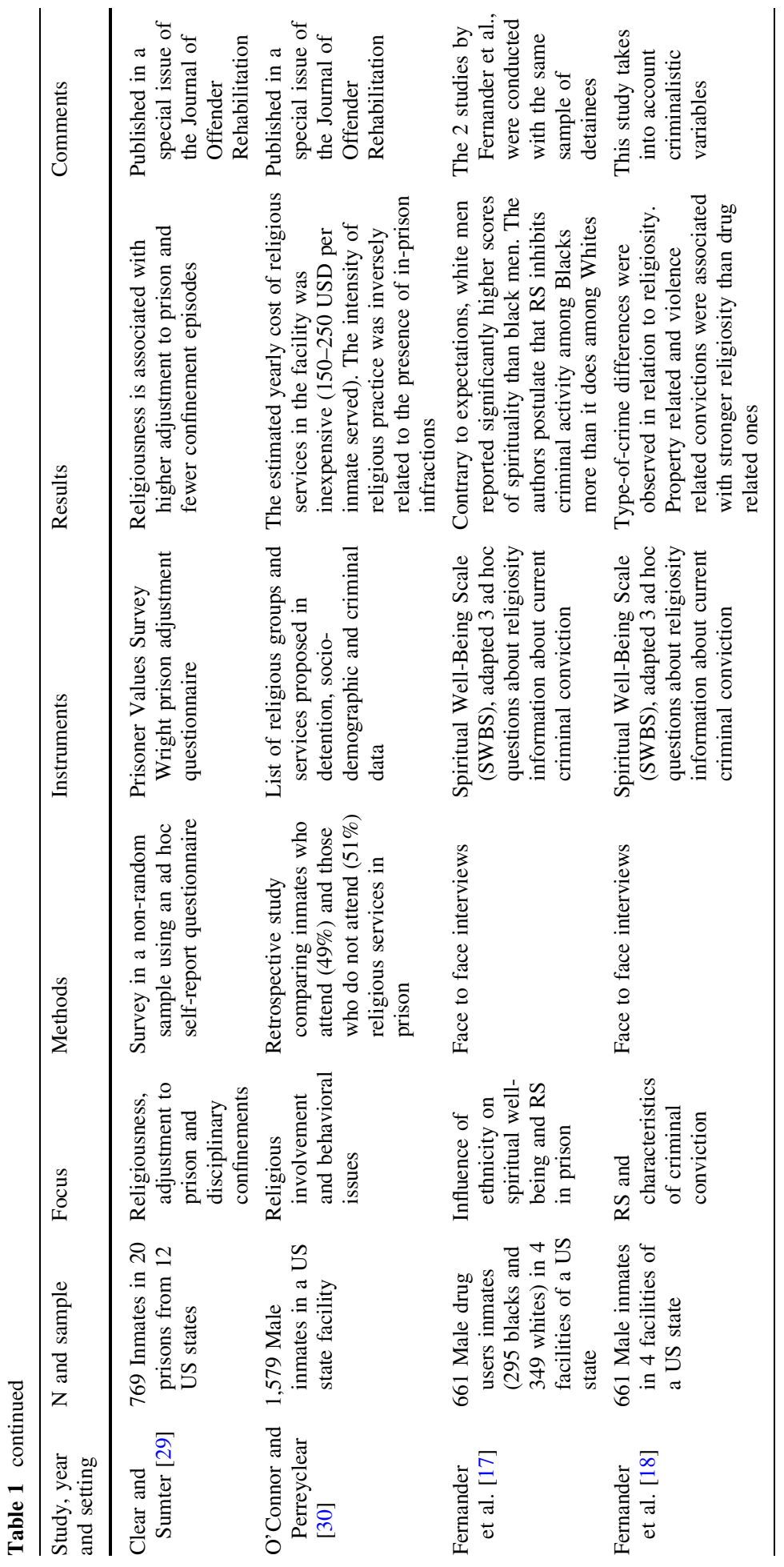




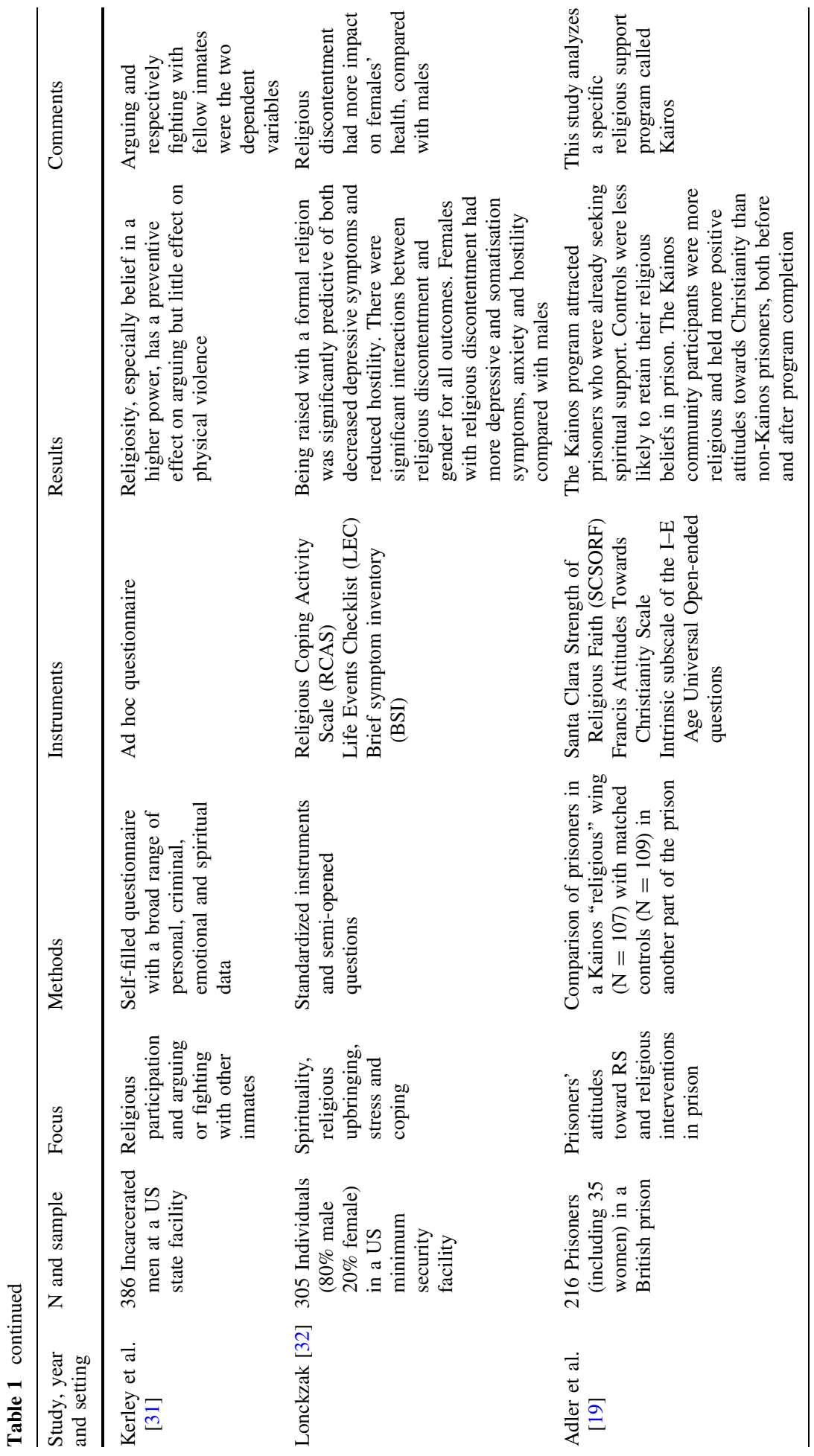




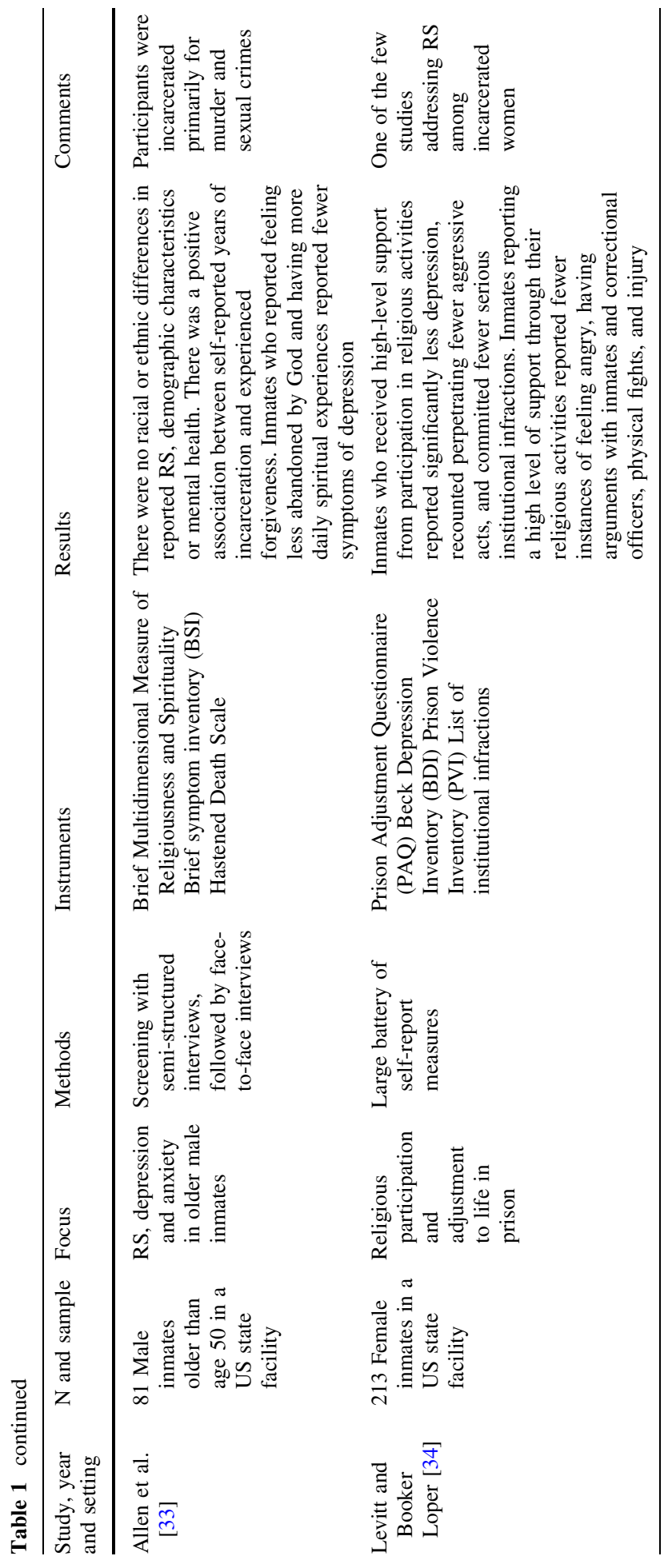




\section{Discussion and Conclusions}

There are little systematic quantitative studies on the impact of RS on the mental health of detained individuals. Due to the variety of psychometric instruments used and the lack of methodological standardization, it is not possible to generalize results of published studies. Moreover, there is a lack of international data and information from other countries than the USA, either form empirical or qualitative studies.

Despite previous knowledge that RS is an important coping mechanism in stressful situations, there is no proof that RS significantly reduces suicide in prison. This could be due to the absence of study addressing specifically this question. From available data, there are however indications showing that RS is associated with lower frequency and severity of depression. The strongest reported effect of RS on prison life is a reduction of incidents and disciplinary sanctions.

\section{References}

1. Walmsley R: World Prison Population List. London, King's College, 2009

2. Andersen HS: Mental health in prison populations. A review-with special emphasis on a study of Danish prisoners on remand. Acta Psychiatrica Scandinavica 424 (Suppl):5-59, 2004.

3. Fazel S, Lubbe S: Prevalence and characteristics of mental disorders in jails and prisons. Current Opinion Psychiatry 18(5):550-554, 2005

4. Fazel S, Danesh J: Serious mental disorder in 23000 prisoners: A systematic review of 62 surveys. Lancet 359(9306):545-550, 2002

5. Assadi SM et al.: Psychiatric morbidity among sentenced prisoners: Prevalence study in Iran. British Journal of Psychiatry 188:159-164, 2006

6. Fatoye FO et al.: Psychological characteristics as correlates of emotional burden in incarcerated offenders in Nigeria. East African Medical Journal 83(10):545-552, 2006

7. Banerjee A, Sengupta P, Ray TK: Persons with major psychiatric illness in prisons: A three years study. Journal of Indian Medical Association. 107(1):14-16, 2009

8. Wortzel HS et al.: Suicide among incarcerated veterans. Journal of the American Academy of Psychiatry and the Law 37(1):82-91, 2009

9. Blaauw E, Kerkhof AJ, Hayes LM: Demographic, criminal, and psychiatric factors related to inmate suicide. Suicide and Life Threatening Behavior 35(1):63-75, 2005

10. Shaw J et al.: Suicide by prisoners, National clinical survey. British Journal of Psychiatry 184:263-267, 2004

11. Daniel AE: Preventing suicide in prison: A collaborative responsibility of administrative, custodial, and clinical staff. Journal of the American Academy of Psychiatry and the Law. 34(2):165-175, 2006

12. Jenkins R et al.: Psychiatric and social aspects of suicidal behaviour in prisons. Psychological Medicine 35(2):257-269, 2005

13. Koenig HG: Research on religion, spirituality, and mental health: A review. Canadian Journal of Psychiatry 54(5):283-291, 2009

14. Mohr S et al.: Toward an integration of spirituality and religiousness into the psychosocial dimension of schizophrenia. American Journal of Psychiatry 163(11):1952-1959, 2006

15. Borras $L$ et al.: The relationship between addiction and religion and its possible implication for care. Substance Use \& Misuse 45(14):2357-410, 2010

16. Koenig HG: Concerns about measuring "spirituality" in research. Journal of Nervous and Mental Disease 196(5):349-355, 2008

17. Fernander A et al.: An exploratory examination of the Spiritual Well-Being Scale among incarcerated black and white male drug users. International Journal of Offender Therapy and Comparative Criminology 48(4):403-413, 2004

18. Fernander A et al.: Exploring the type-of-crime hypothesis, religiosity, and spirituality in an adult male prison population. International Journal of Offender Therapy and Comparative Criminology 49(6):682-695, 2005

19. Adler JR et al.: Measuring religion in prisons: Offenders' beliefs and attitudes. Journal of Forensic Psychology Practice 8(2):130-149, 2008 
20. Kerley KR, Copes H: "Keepin' my mind right” identity maintenance and religious social support in the prison context. International Journal of Offender Therapy and Comparative Criminology 53(2): 228-244, 2009

21. Dix-Richardson F.: Resistance to conversion to Islam among African American women inmates. Journal of Offender Rehabilitation 35(3-4):109-126, 2002

22. Schneider RZ, Feltey KM: "No matter what has been done wrong can always be redone right": Spirituality in the lives of imprisoned battered women. Violence Against Women 15(4):443-459, 2009

23. Dammer HR: The reasons for religious involvement in the correctional environment. Journal of Offender Rehabilitation 35(3-4):35-58, 2002

24. Skotnicki A: Evaluating religious initiatives in a correctional setting: Do inmates speak? Journal of Offender Rehabilitation 35(3-4):199-214, 2002

25. Enache A et al.: Aspects regarding the hope for successful reintegration of female detainees. Legal Medicine (Tokyo). 11(Suppl 1):S382-S385, 2009

26. Scarnati $\mathrm{R}$ et al.: Religious beliefs and practices among most-dangerous psychiatric inmates. Forensic Reports 4(1):1-16, 1991

27. Koenig HG: Religion and older men in prison. International Journal of Geriatric Psychiatry 10(3):219-230, 1995

28. Pass MG: Religious orientation and self-reported rule violations in a maximum security prison. Journal of Offender Rehabilitation 28(3-4):119-134, 1999

29. Clear TR, Sumter MT: Prisoners, prison, and religion: Religion and adjustment to prison. Journal of Offender Rehabilitation 35(3-4):127-159, 2002

30. O'Connor TP, Perreyclear M: Prison religion in action and its influence on offender rehabilitation. Journal of Offender Rehabilitation 35(3-4):11-33, 2002

31. Kerley KR, Matthews TL, Blanchard TC: Religiosity, religious participation, and negative prison behaviors. Journal for the Scientific Study of Religion 44(4):443-457, 2005

32. Lonczak HS et al.: Religious coping and psychological functioning in a correctional population. Mental Health, Religion \& Culture 9(2):171-192, 2006

33. Allen RS et al.: Religiousness/spirituality and mental health among older male inmates. Gerontologist 48(5):692-697, 2008

34. Levitt L, Loper AB: The influence of religious participation on the adjustment of female inmates. American Journal of Orthopsychiatry 79(1):1-7, 2009

\section{Author Biography}

Ariel Eytan, MD is a specialist in psychiatry and psychotherapy FMH. He is the head of the Division of Penitentiary Psychiatry at the University Hospitals of Geneva, Switzerland. He is Privat-docent at the Faculty of Medicine, Department of Psychiatry. 